\title{
Point-of-care tests for STIs: the way forward
}

\author{
Igor Toskin, ${ }^{1}$ Rosanna W Peeling, ${ }^{2}$ David Mabey, ${ }^{3}$ King Holmes, ${ }^{4}$ \\ Ronald Ballard, ${ }^{1}$ James Kiarie, ${ }^{1}$ lan Askew ${ }^{1}$
}

It has been over 10 years since the WHO Sexually Transmitted Diseases Diagnostics Initiative (SDI) published a full supplement in this journal on rapid diagnostic testing for STIs, ${ }^{1}$ making the case for quality-assured rapid STI tests to help countries achieve their Millennium Development Goals and decrease the STI burden worldwide. The supplement introduced the ASSURED criteria to assess whether diagnostics were appropriate for disease control needs, namely: Affordable, Sensitive, Specific, User-friendly, Rapid and robust, Equipment-free and Deliverable to end users. ${ }^{2}$ This ASSURED approach has broadened the paradigm of STI testing from rapid to point-of-care testing (POCT) or near-patient testing. Testing and delivering the results rapidly and conveniently to the patient will increase the likelihood of appropriate and immediate treatment and decrease the chances of onward transmission and development of reproductive sequelae.

The most recent WHO estimates found that in 2012, among people aged 15-49 years, there were an estimated 6 million new cases of syphilis each year, 78 million cases of gonorrhoea, 131 million cases of chlamydial infection and 142 million cases of trichomoniasis-the four most common curable STIs. ${ }^{3}$ A separate literature review, including a modelling analysis, suggested that in 2012, 417 million people 15-49 years of age were living with herpes simplex virus type 2 infection worldwide, ${ }^{4}$ and 291 million women with genital human papillomavirus (HPV) infection. ${ }^{5}$ These pandemics, and the growing number of STIs caused by other pathogens have a profound impact on the health and lives of children, adolescents and adults worldwide. ${ }^{6}$

\footnotetext{
'Department of Reproductive Health and Research, World Health Organization, Geneva, Switzerland ${ }^{2}$ Department of Clinical Research, London School of Hygiene and Tropical Medicine, London, UK ${ }^{3}$ Department of Infectious and Tropical Diseases, London School of Hygiene and Tropical Medicine, London, UK

${ }^{4}$ Department of Global Health \& Medicine, University of Washington, Seattle, Washington, USA
}

Correspondence to Dr Igor Toskin, Department of Reproductive Health and Research, World Health Organization, Geneva, Switzerland; toskini@who.int
To achieve a sustainable decrease of STI infections globally, the new WHO STI health sector strategy reaffirms the need for early diagnosis of STIs, screening for asymptomatic carriers of infection and linkage to treatment, as a core intervention. ${ }^{6}$ The strategy nevertheless acknowledges the lack of reliable, low-cost POCTs as a major barrier to advancing STI control and prevention, and lists the validation and standardisation of innovative diagnostic technologies and approaches as one of the key priorities.

Since the supplement on rapid tests for STIs in 2006, considerable progress has been made in POCT technology, conceptualisation, evaluation and implementation, but this has not yet been translated into global uptake of POCTs as one of the essential interventions included in public health strategies. Therefore, the WHO Department of Reproductive Health and Research convened two technical consultations, in 2014 and 2015, with experts from all regions, to discuss the state of affairs in the POCT field, defining the gaps and the way forward. The current supplement offers insight into the evolution of POCT in the last 10 years and the plans for the future.

The first article in this supplement gives an overview of target product profiles, as defined during the expert consultation, against which the currently available POCTs can be assessed, and should help to guide the development and validation of new STI POCTs. It also includes a summary of the current technologies and tests on the market for the four major curable STIs, and for HPV, as well as POCTs that are in the pipeline. In the 2006 supplement, several papers described performance characteristics of simple lateral flow devices. During the 10 years following its publication, research groups have developed more sophisticated technologies, some based on molecular testing. Looking forward, progress in microfluidics and nanotechnology will hopefully allow for multiplex POC platforms and enable quantitative analyses. ${ }^{7}$

POCTs are promising tools to combat the increasing threat of antimicrobial resistance (AMR) in both STI pathogens and other microorganisms. ${ }^{8}$ In this supplement, new POCT technologies are discussed that enable AMR sensitive diagnosis. This will drastically reduce inappropriate prescribing and dispensing of antibiotics, which is indispensable for global strategies to control the spread and impact of AMR. ${ }^{9}$

POCTs may have excellent performance characteristics in a laboratory environment, but their usefulness, their performance and operational characteristics, should be assessed in clinical settings and in the field. This supplement includes systematic reviews that synthesise the results of field evaluations of POCTs for chlamydial infection, gonorrhoea, trichomoniasis and HPV infection; and also summarises performance of dual HIV/ syphilis POCTs in different patient groups and clinical settings.

POCTs should increasingly become the diagnostics of the future, particularly in low and middle-income countries, both in primary care centres and outreach settings, enabling patient-centred STI testing and treatment in key populations. ${ }^{10}{ }^{11}$ However, our systematic reviews showed that we need improvements in the sensitivity of antigen detection lateral flow assays and more affordable (near) POC molecular assays before necessary guidelines and recommendations can be made on the use of POCTs in low resource settings, both for screening programmes for detection of asymptomatic infections and to complement syndromic management algorithms in order to guide better clinical decisions and follow-up. The potential impact of POCTs in regions where syndromic management is the standard of care cannot be overemphasised.

The potential use of syphilis POCTs in reaching vulnerable populations such as the indigenous population in the Brazilian Amazon, is an important element of the global control strategy. ${ }^{6}$ Our supplement offers an example on the programmatic potential of POCTs in reducing the burden of syphilis in men who have sex with men in Italy.

The introduction of POC CD4 enumeration technologies has shown that managing a national network of POC sites can be challenging in some contexts. ${ }^{12}$ Decentralised testing requires major commitments to training and monitoring of effectiveness, while ensuring quality test results and avoiding operator and instrument errors, instrument breakdowns, and stock-outs of reagents or other supplies. Technological innovation alone will thus not be sufficient. Decentralisation of testing could be seen as a concern 
by laboratories, as some of their tasks regarding STI testing, might be diminished. Our international expert group suggested anticipating these tensions by assessing the capability and willingness of laboratories to take up their new role, that is, to provide training, quality assurance, and monitor and evaluate STI testing, during and after the shift to POCTs. The laboratory will become both a command and training centre for POCTs and its providers and a centre of quality assurance and certification. ${ }^{13}$

During the technical consultations, experts did point to the knowledge gaps in basic research and development of POCTs, and discussed their evaluation and potential programmatic impact. Suggestions to advance the field of POCTs are presented in the last article of the supplement. The 'roadmap' elaborates more on decentralised testing and the new important role of reference laboratories, the surveillance potential of using connectivity technologies, self-testing, and the need for an investment case. It also reflects on the assessment of the needs of and feedback ${ }^{14}$ from end users, both patients and healthcare providers, which are crucial for successful roll-out of POCTs. This is especially important for diagnostic tools which can be used as self-tests.

If implemented sensibly, POCTs can be catalysts for health systems strengthening globally. ${ }^{14} 15$ The health sector response to STI epidemics is critical to the achievement of universal health coverage-one of the key targets of the Sustainable Development Goals. POCT could in the long run replace non-specific syndromic management of STIs with specific recommended treatments for proven infections. POCTs also have the potential of expanding targeted STI screening and rapidly linking the nfected patients to treatment. This may decrease STI incidence, and consequent morbidity and mortality, improving the sexual health and well-being of all people.

Contributors All authors defined the concept and content of the editorial, IT wrote the manuscript; all authors reviewed, edited and approved the editorial.

Disclaimer The authors are staff members of the World Health Organization. The authors alone are responsible for the views expressed in this publication and they do not necessarily represent the views, decisions or policies of the World Health Organization.

Competing interests None declared.

Provenance and peer review Commissioned; externally peer reviewed.

\section{(1) \\ OPEN ACCESS}

(c) World Health Organization 2017. Licensee BMJ Publishing Group Limited. This is an open access article distributed under the terms of the Creative Commons Attribution IGO License (https://creativecommons.org/ licenses/by/4.0/igo), which permits use, distribution, and reproduction for non-commercial purposes in any medium, provided the original work is properly cited. In any reproduction of this article there should not be any suggestion that WHO or this article endorse any specific organization or products. The use of the WHO logo is not permitted. This notice should be preserved along with the article's original URL.

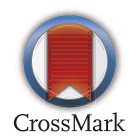

To cite Toskin I, Peeling RW, Mabey D, et al. Sex Transm Infect 2017;93:S1-S2.

Received 3 April 2017

Revised 21 June 2017

Accepted 2 July 2017

Sex Transm Infect 2017:93:S1-S2.

doi:10.1136/sextrans-2016-053074

\section{REFERENCES}

1 Peeling RW, Holmes KK, Mabey D, et al. Rapid tests for sexually transmitted infections (STIS): the way forward. Sex Transm Infect 2006;82(Suppl V):v1-6.
2 Peeling RW, Mabey D, Herring A, et al. Why do we need quality-assured diagnostic tests for sexually transmitted infections? Nat Rev Microbiol 2006:4:909-21.

3 Newman L, Rowley J, Vander Hoorn S, et al. Global estimates of the prevalence and incidence of four curable sexually transmitted infections in 2012 based on systematic review and global reporting. PLoS One 2015; 10:e0143304.

4 Looker KJ, Magaret AS, Turner KM, et al. Global estimates of prevalent and incident herpes simplex virus type 2 infections in 2012. PLoS One 2015;10:e114989.

5 de Sanjosé S, Diaz M, Castellsagué $X$, et al. Worldwide prevalence and genotype distribution of cervical human papillomavirus DNA in women with normal cytology: a meta-analysis. Lancet Infect Dis 2007;7:453-9.

6 World Health Organization. Global health sector strategy on sexually transmitted infections, 2016-2021. Geneva:WHO, 2016. http://www.who. int/reproductivehealth/publications/rtis/ghss-stis/en/.

7 Damhorst GL, Murtagh M, Rodriguez WR, et al. Microfluidics and nanotechnology for detection of global infectious diseases. Proc IEEE Inst Electr Electron Eng 2015;103:150-60.

8 World Health Organization. Global action plan on antimicrobial resistance. Geneva:WHO, 2015. http:// www.who.int/antimicrobial-resistance/publications/ global-action-plan/en/

9 World Health Organization. Global action plan to control the spread and impact of antimicrobial resistance in Neisseria gonnorrhoeae. Geneva:WHO 2012. http://www.who.int/reproductivehealth/ publications/rtis/9789241503501/en/

10 World Health Organization. Report on global sexually transmitted infection surveillance 2013. Geneva: WHO, 2014. http://www.who.int/reproductivehealth/ publications/rtis/stis-surveillance-2013/en/

11 Garcia PJ, You P, Fridley G, et al. Point-of-care diagnostic tests for low-resource settings. Lancet Glob Health 2015;3:e257-8.

12 Nkengasong J, Boeras DI, Abimiku Alash'le, et al. Assuring the quality of diagnostic testing: The future is now. Afr J Lab Med 2016;5:a558.

13 Boeras DI, Nkengasong JN, Peeling RW. Implementation science: the laboratory as a command centre. Curr Opin HIV AIDS 2017;12:171-4.

14 Mabey DC, Sollis KA, Kelly HA, et al. Point-of-care tests to strengthen health systems and save newborn lives: the case of syphilis. PLoS Med 2012:9:e1001233.

15 García PJ, Cárcamo CP, Chiappe M, et al. Rapid syphilis tests as catalysts for health systems strengthening: a case study from Peru. PLoS One 2013;8:e66905. 